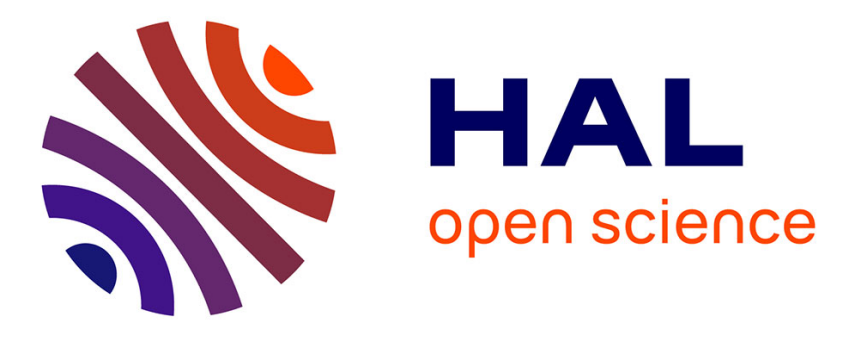

\title{
Optimal Location of Actuators and Sensors in Active Vibration Control
}

\author{
Isabelle Bruant, Laurent Proslier
}

\section{To cite this version:}

Isabelle Bruant, Laurent Proslier. Optimal Location of Actuators and Sensors in Active Vibration Control. Journal of Intelligent Material Systems and Structures, 2016, 16 (3), pp.197-206. 10.1177/1045389X05047989 . hal-01978500

\section{HAL Id: hal-01978500 \\ https://hal.parisnanterre.fr/hal-01978500}

Submitted on 11 Jan 2019

HAL is a multi-disciplinary open access archive for the deposit and dissemination of scientific research documents, whether they are published or not. The documents may come from teaching and research institutions in France or abroad, or from public or private research centers.
L'archive ouverte pluridisciplinaire $\mathbf{H A L}$, est destinée au dépôt et à la diffusion de documents scientifiques de niveau recherche, publiés ou non, émanant des établissements d'enseignement et de recherche français ou étrangers, des laboratoires publics ou privés. 


\title{
Optimal Location of Actuators and Sensors in Active Vibration Control
}

\author{
Bruant I., Proslier L. \\ Laboratoire de Mécanique de Paris X, \\ 1 Chemin Desvallières, \\ 92410 Ville d'Avray, France
}

\begin{abstract}
Several studies have been developed in order to find the optimal location of actuators and sensors in active control of structures. In this paper, a modified optimization criteria is proposed for these two optimization problems, ensuring good observability or good controllability of the structure, and considering residual modes to limit the spillover effects. Its efficiency is shown by comparison with classical criteria, illustrated for a simply supported beam and a rectangular plate. In these two applications, the number of active elements is discussed, using or neglecting the residual modes.
\end{abstract}

Keywords: Active Vibration Control, Optimal location and number, actuators and sensors, numerical results

\section{INTRODUCTION}

In vibration suppression of structures, some parameters, such as location of actuators and sensors, have a major influence on the performance of the control system. It is well known that misplaced sensors and actuators lead to problems such as the lack of observability or controllability. In this way, different cost functions have been used to find the optimal locations of these active elements.

In the case of optimization of actuators locations,(Arbel, 1981), (Hac and Liu, 1993) and (Devasia et al., 1993) proposed to maximize a controllability criteria using a measure of the gramian matrix. This approach seeks to ensure active damping of all needed modes by minimizing the control energy. In a second approach (Wang and Wang, 2001) suggested to maximize the control forces transmitted by the actuators to the structure. A third optimization cost function is a linear quadratic optimal framework. (Dhingra and Lee, 1995), (Kondoh et al., 1990), (Bruant et al., 2001) and (Yang and Lee, 1993) proposed a quadratic cost function taking into account the measurement error and control energy. In the case of sensors locations, the most usual performance uses the energy of the state output so as to maximize the information given by sensors. (Baruh, 1992) and (Hac and Liu, 1993) rather proposed to maximize measures of the gramian observability matrix in order to have optima without dependence on initial conditions.

In this paper, a methodology is presented to find the actuators and sensors location to increase control quality. In order to simplify the optimization problem, it was decided to search independently the optimal location of actuators and that of sensors.

In applications, the external disturbances are not allways wellknown. In addition, many regulator methods can be used. Then, it seems to be better to define an optimization methodology independent of these external work parameters. From these reasons, we propose here to obtain optimal actuators location by maximizing a modified criteria which can result from the two first different approaches. From a given number of eigenmodes, our objective is to find the actuators locations such that the actuators control is the largest possible using less possible electrical energy. In the same way, as the sensors have to inform the control system about the vibrationnal state of the structure for each eigenmode, we present a modified criteria in order to maintain the system output as large as possible.

The spillover effects appear as a strong problem of active control implementation on real structures. Very few papers take into account the residual modes in their developpements (Gaudiller and Hagopian, 1996), (Collet, 1999). Here, each criteria is presented considering them, in order to limit the actuation or observation by active elements on a certain subset of modes.

I. Bruant: E-mail: isabelle.bruant@u-paris10.fr 
In section 2 , we point out the active vibration control equations. In section 3 and 4 , we present the optimization criteria used for sensors and actuators location. Comparisons with classical criteria are shown in section 5 , illustrated for a simply supported plate with piezoelectric active elements. Discussion is developed about the optimal number of actuators and sensors, especially when residual modes are taken into account in the cost functions. Results are shown for a simply supported beam with point sensors.

\section{EQUATIONS OF ACTIVE CONTROL}

Consider a flexible structure with $N_{a}$ actuators and $N_{s}$ velocity sensors (all further developpements can be applied for displacement sensors). The motion's equations and the sensors' equations of the system in modal coordinates can be written as follows, when the $N$ first eigenmodes are considered and when taking into account the $N_{R}$ residual eigenmodes:

$$
\begin{gathered}
\ddot{\alpha}_{i}+2 \zeta_{i} \omega_{i} \dot{\alpha}_{i}+\omega_{i}^{2} \alpha_{i}=\sum_{l=1}^{N_{a}} b_{i l} \Phi_{l} \quad i=1 . . N \\
\ddot{\alpha}_{i}+2 \zeta_{i}^{R} \omega_{i}^{R} \dot{\alpha}_{i}+\left(\omega_{i}^{R}\right)^{2} \alpha_{i}=\sum_{l=1}^{N_{a}} b_{i l}^{R} \Phi_{l} \quad i=1 . . N^{R} \\
y_{j}=\sum_{l=1}^{N} c_{j l} \dot{\alpha}_{l}+\sum_{l=1}^{N^{R}} c_{j l}^{R} \dot{\alpha}_{l} \quad j=1 . . N_{s}
\end{gathered}
$$

$\alpha_{i}, \dot{\alpha}_{i}$ and $\ddot{\alpha}_{i}$ represent modal displacement, velocity and acceleration, $\omega_{i}$ and $\zeta_{i}$ are the natural frequency and damping ratio of the ith mode, and $\omega_{i}^{R}$ and $\zeta_{i}^{R}$ those of the residual modes; $b_{i l} \Phi_{l}$ is the ith modal component of the control force due to $\Phi_{l}$ applied to the actuator $l, b_{i l}^{R} \Phi_{l}$ is the ith residual modal component of the force appearing with the actuation of the actuator $l . y_{j}$ is the quantity measured from the jth sensor. $c_{j l}$ is the sensing constant of the jth sensor due to the motion of the lth mode and $c_{j l}^{R}$ those due to the motion of the lth residual mode. $b_{i l}, b_{i l}^{R}$, $c_{j l}, c_{j l}^{R}$ depend respectively of the lth actuator location and jth sensor location.

These equations can be written in a state-space form, using the state vector $\{x\}\left(\operatorname{size}\left(N+N^{R}\right)+\left(N+N^{R}\right)\right)$ :

$$
\begin{gathered}
\{x\}=\left\{\omega_{i} \alpha_{i} \quad \dot{\alpha}_{i}\right\}^{T} \\
\frac{d}{d t}\{x\}=[A]\{x\}+[B]\{\Phi\} \\
\{y\}=[C]\{x\}
\end{gathered}
$$

$[A]_{\left(2 N+2 N^{R}, 2 N+2 N^{R}\right)},[B]_{\left(2 N+2 N^{R}, N_{a}\right)}$ and $[C]_{\left(N_{s}, 2 N+2 N^{R}\right)}$ are the state, control and output matrices given by:

$$
\begin{aligned}
& {[A]=\left[\begin{array}{cccc}
{[0]} & {[0]} & {\left[\omega_{i}\right]} & {[0]} \\
{[0]} & {[0]} & {[0]} & {\left[\omega_{i}^{R}\right]} \\
-\left[\omega_{i}\right] & {[0]} & -\left[2 \zeta_{i} \omega_{i}\right] & {[0]} \\
{[0]} & -\left[\omega_{i}^{R}\right] & {[0]} & -\left[2 \zeta_{i}^{R} \omega_{i}^{R}\right]
\end{array}\right]} \\
& {[B]=\left[\begin{array}{c}
{[0]} \\
{\left[b_{i l}\right]} \\
{[0]} \\
{\left[b_{i l}^{R}\right]}
\end{array}\right]}
\end{aligned}
$$

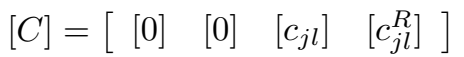

$\Phi$ is the actuation vector: in the case of piezoelectric actuators, it contains electrical potentials.

From equation (4), several automatic tools can be used to actively control vibrations (Preumont, 1999) by applying a well chosen $\{\Phi\}$. The actuation must be defined to bend the $N$ first eigenmodes, but it also actuates the residual modes exciting them. This effect is called spillover. In fact, the best case would be having:

$$
\begin{gathered}
b_{i l}>>0 \quad \forall l \in 1, \ldots N_{a} \quad \text { and } \quad \forall i \in 1 \ldots N \\
b_{i l}^{R}=0 \quad \forall l \in 1, \ldots N_{a} \quad \text { and } \quad \forall i \in 1 \ldots N^{R},
\end{gathered}
$$

ensuring actuation (or controllability) for the $N$ first modes and no influence (or non controllability) of the $N^{R}$ residual modes. This configuration should be approached by optimizing the actuators locations.

In the same way, the vibrational information given by the output sensors to the control system contains motions on residual modes. As the control system neglects these residual modes, the received information does not correspond to the needed information. The best case would be having:

$$
\begin{gathered}
c_{j l}>>0 \quad \forall j \in 1, \ldots N_{s} \quad \text { and } \quad \forall l \in 1 \ldots N \\
c_{j l}^{R}=0 \quad \forall j \in 1, \ldots N_{s} \quad \text { and } \quad \forall l \in 1 \ldots N^{R},
\end{gathered}
$$

in order to have each mode $(l \in 1 \ldots N)$ well observed (good observability) and each residual mode not observed (non observability). As before, this configuration should be approached by optimizing the sensors locations.

Hence, before setting up the regulator and observer system, the active elements' locations have to be defined. 


\section{OPTIMAL LOCATION OF ACTUATORS}

Many studies have been developed on optimal location of actuators. Two usual approaches are used before setting up the regulator: minimizing the control energy required by maximizing a measure of the controllability gramian matrix, and maximizing in a certain way the control force.

In this work, we suggest considering these two approaches: finding the actuators' locations so that the actuators' forces are the largest possible (2nd approach) using electrical energy (1st approach) as possible.

In the next subsections, we briefly describe these approaches and explain our modified criteria.

\section{Minimization of the control energy}

This method is detailed in (Hac and Liu, 1993). The objective here is to find actuators locations which minimize the control energy required to bring the modal system (considering the $N$ first eigenmodes) to a desired state $\left\{x_{T}\right\}$ after some time $T$ :

$$
J=\min _{\{\Phi\}} \int_{0}^{T}\{\Phi\}^{T}\{\Phi\} d t
$$

The optimal solution gives the following optimal control energy :

$$
J=\left[e^{[A] T}\left\{x_{0}\right\}-\left\{x_{T}\right\}\right]^{T} W^{-1}(T)\left[e^{[A] T}\left\{x_{0}\right\}-\left\{x_{T}\right\}\right.
$$

where $W(T)$ is the controllability gramian matrix defined by :

$$
W(T)=\int_{0}^{T} e^{[A] t}[B][B]^{T} e^{[A]^{T} t} d t
$$

Hence, as the actuators' effects are only in $W(T)$ from $[B]$, minimizing $J$ with respect to the actuators locations consists in minimizing $W^{-1}(T)$ or maximizing a measure of the controllability gramian matrix.

(Hac and Liu, 1993) has shown that instead of using $W(T)$, a steady state $W_{c}$ can be considered to eliminate the dependency of the solution $T$. $W_{c}$ tends to a diagonal form with

$$
\begin{aligned}
& \left(W_{c}\right)_{i i}=\left(W_{c}\right)_{i+N+N^{R}, i+N+N^{R}}=\left(W_{c}^{U}\right)_{i i} \\
& =\sum_{j=1}^{N_{a}} \frac{b_{i j}^{2}}{4 \zeta_{i} \omega_{i}}=\frac{1}{4 \zeta_{i} \omega_{i}} \sum_{j=1}^{N_{a}} b_{i j}^{2} \\
& \left(W_{c}\right)_{i+N, i+N}=\left(W_{c}\right)_{i+2 N+N^{R}, i+2 N+N^{R}}=\left(W_{c}^{R}\right)_{i i} \\
& =\sum_{j=1}^{N_{a}} \frac{\left(b_{i j}^{R}\right)^{2}}{4 \zeta_{i}^{R} \omega_{i}^{R}} \quad i=1, \ldots N^{R}
\end{aligned}
$$

$$
=\frac{1}{4 \zeta_{i}^{R} \omega_{i}^{R}} \sum_{j=1}^{N_{a}}\left(b_{i j}^{R}\right)^{2} \quad i=1, \ldots N^{R}
$$

$\left(W_{c}^{U}\right)_{i i}$ and $\left(W_{c}^{R}\right)_{i i}$ equal to the energy transmitted from the actuators to the structure for the ith used and residual eigenmode.

Hence if one eigenvalue of $\left(W_{c}^{U}\right)_{i i}$ is small there is an eigenmode that is difficult to control: there is no controllability for the system. Similarly, if one eigenvalue $\left(W_{c}\right)_{i i}^{R}$ corresponding to a residual mode is high, the induced spillover effect can be important. In order to avoid these cases, several criteria can be maximized, as (Arbel, 1981), (Hac and Liu, 1993), (Collet, 1999):

$$
\operatorname{det}\left(W_{c}^{U}\right)-\gamma \operatorname{det}\left(W_{c}^{R}\right)
$$

$\operatorname{trace}\left(W_{c}^{U}\right) *\left(\operatorname{det}\left(W_{c}^{U}\right)\right)^{1 / 2 N}-\gamma \operatorname{trace}\left(W_{c}^{R}\right) *\left(\operatorname{det}\left(W_{c}^{R}\right)\right)^{1 / 2 N}$

$$
\min _{i=1, N}\left(W_{c}^{U}\right)_{i i}-\gamma \max _{i=1, N^{R}}\left(W_{c}^{R}\right)_{i i}
$$

where $\gamma$ is a weighting constant. These three criteria ensure global controllability of the system for the $N$ first eigenmodes and try to minimize the global excitation of the $N^{R}$ residual modes.

\section{Maximization of the control force}

In this approach, detailed in (Wang and Wang, 2001), the actuators locations are obtained by maximizing the global control force $\left\{f_{c}\right\}=[B]\{f\}$ from $\left\{f_{c}\right\}^{T}\left\{f_{c}\right\}=$ $\{\Phi\}^{T}[B]^{T}[B]\{\Phi\}$ (here residual modes are neglected).

Using singular value analysis, $[B]$ can be written as: $[B]=[M][S][N]^{T}$ where $[M]^{T}[M]=[I]=[N]^{T}[N]$,

$$
[S]=\left[\begin{array}{ccc}
\sigma_{1} & 0 & 0 \\
0 & \sigma_{i} & 0 \\
0 & 0 & \sigma_{N_{a}} \\
0 & 0 & 0
\end{array}\right]
$$

and

$$
\left\{f_{c}\right\}^{T}\left\{f_{c}\right\}=\{\Phi\}^{T}[N][S]^{T}[S][N]^{T}\{\Phi\}
$$

or $\left\|\left\{f_{c}\right\}\right\|^{2}=\|\{\Phi\}\|^{2}\|S\|^{2}$. Thus, maximizing this norm independently on the input $\{\Phi\}$ induces maximizing $\|S\|^{2}$. Wang suggests to maximize

$$
\prod_{i=1}^{N_{a}} \sigma_{i}^{2}
$$

taking into account globally all modes. In this equation, $\sigma_{i}$ is refered to as the ith degree of controllability of the system. Its magnitude is a function of the location and size of the piezoelectric actuators. 
When residual modes cannot be neglected, a similar criteria can be obtained, consisting in differentiating the $N$ first modes from the $N_{R}$ residual modes and maximizing :

$$
\prod_{i=1}^{N_{a}} \sigma_{i}^{2}-\gamma \prod_{i=1}^{N_{a}}\left(\sigma_{i}^{R}\right)^{2}
$$

where $\sigma_{i}^{R}$ are the components of $[S]^{R}$ corresponding to $b^{R}$, and $\gamma$ is a weighting constant.

\section{The modified criteria}

The use of these criteria shows that the eigenmodes are taken into account globally. As our objective is to control each considered modes, without exciting residual modes, by transmitting a maximum control force with a minimum electrical energy, we suggest here modifying these criteria.

- First of all, instead of maximizing a global norm of $W_{c}$ which means minimizing the electrical energy, we suggest maximizing each diagonal term of $W_{c}$ (the energy transmitted from the actuators to the structure for each eigenmode $(1 \ldots N)$ ) and minimizing the $N^{R}$ other diagonal term of $W_{c}$ :

$$
\begin{aligned}
\text { maximize } & \left\{\left(W_{c}^{U}\right)_{11}, \ldots,\left(W_{c}^{U}\right)_{N N}\right\} \\
\text { minimize } & \left\{\left(W_{c}^{R}\right)_{11}, \ldots,\left(W_{c}^{R}\right)_{N^{R} N^{R}}\right\}
\end{aligned}
$$

This optimization problem has $N_{a}$ optimization parameters (the locations of the $N_{a}$ actuators) and $N+N^{R}$ optimization functions. It can be simplified by considering the new optimization criteria:

$$
\text { maximize }\left\{\min _{i=1, \ldots N}\left(W_{c}^{U}\right)_{i i}-\gamma \max _{i=1, \ldots N^{R}}\left(W_{c}^{R}\right)_{i i}\right\}
$$

where $\gamma$ is a weighting constant. This criteria, with $\gamma=0$ has been proposed by (Arbel, 1981), but as the components of $W_{c}$ have not the same range, solving this problem can induce the study of particular modes instead of each of them, and then the obtained locations are not optimal.

Consequently, we decide to establish homogeneity between each term $\left(W_{c}\right)_{i i}$ by dividing each of them by its maximal value obtained if the ith mode is the specific mode to be controlled. This maximal value is the maximal energy which can be transmitted from the actuators for the ith eigenmode.

Hence, the optimization problem becomes, by using the homogeneous components: maximizing

$$
\left\{\min _{i=1, N} \frac{\left(W_{c}^{U}\right)_{i i}}{\max _{\text {locations }}\left(W_{c}^{U}\right)_{i i}}-\gamma \max _{i=1, N^{R}} \frac{\left(W_{c}^{R}\right)_{i i}}{\max _{\text {locations }}\left(W_{c}^{R}\right)_{i i}}\right\}
$$

$$
=\min _{i=1, N} \frac{\sum_{j=1}^{N_{a}} b_{i j}^{2}}{\max _{\text {locations }} \sum_{j=1}^{N_{a}} b_{i j}^{2}}-\gamma \max _{i=1, N^{R}} \frac{\sum_{j=1}^{N_{a}}\left(b_{i j}^{R}\right)^{2}}{\max _{\text {locations }} \sum_{j=1}^{N_{a}}\left(b_{i j}^{R}\right)^{2}}
$$

and,

$$
\forall i=1, \ldots N+N^{R} \quad 0 \leq \frac{\left(W_{c}\right)_{i i}}{\max _{\text {locations }}\left(W_{c}\right)_{i i}} \leq 1
$$

- In the same way, instead of maximizing the norm of the global control force for the considered modes, we suggest to maximize the applied force for each mode $(1, \ldots N)$ independently of $\{\Phi\}$ and to minimize the applied force for residual modes. For the ith mode, the modal equation can be written in the state space form :

$$
\left[\begin{array}{c}
\omega_{i} \dot{\alpha}_{i} \\
\ddot{\alpha}_{i}
\end{array}\right]=\left[\begin{array}{cc}
0 & \omega_{i} \\
-\omega_{i} & -2 \zeta_{i} \omega_{i}
\end{array}\right]\left[\begin{array}{c}
\omega_{i} \alpha_{i} \\
\dot{\alpha}_{i}
\end{array}\right]+\left[\begin{array}{c}
\{0\} \\
\left\{B_{i}\right\}
\end{array}\right]\{\Phi\}
$$

and $\left\|\left\{f_{c}\right\}\right\|^{2}=\|\{\Phi\}\|^{2} \cdot\left\|S_{i}\right\|^{2}$ where $S_{i}^{2}$ has only a non zero term :

$$
\bar{\sigma}_{i}^{2}=\sum_{j=1}^{N_{a}} b_{i j}^{2}
$$

Then, the optimization problem is:

$$
\text { maximize } \quad\left\{\left\|S_{1}\right\|^{2}, \ldots\left\|S_{N}\right\|^{2}\right\}=\left\{\bar{\sigma}_{1}^{2}, \ldots \bar{\sigma}_{N}^{2}\right\}
$$

and $\operatorname{minimize} \quad\left\{\left\|S_{1}^{R}\right\|^{2}, \ldots\left\|S_{N}^{R}\right\|^{2}\right\}=\left\{\bar{\sigma}_{1}^{2}, \ldots \bar{\sigma}_{N^{R}}^{2}\right\}$

From the same arguments than before, this problem is thus transformed: maximizing

$$
\begin{aligned}
& \left\{\min _{i=1, N} \frac{\bar{\sigma}_{i}^{2}}{\max _{\text {locations }} \bar{\sigma}_{i}^{2}}-\gamma \max _{i=1, N^{R}} \frac{\bar{\sigma}_{i}^{2}}{\max _{\text {locations }} \bar{\sigma}_{i}^{2}}\right\} \\
= & \min _{i=1, N} \frac{\sum_{j=1}^{N_{a}} b_{i j}^{2}}{\max _{\text {locations }} \sum_{j=1}^{N_{a}} b_{i j}^{2}}-\gamma \max _{i=1, N^{R}} \frac{\sum_{j=1}^{N_{a}}\left(b_{i j}^{R}\right)^{2}}{\max _{\text {locations }} \sum_{j=1}^{N_{a}}\left(b_{i j}^{R}\right)^{2}}
\end{aligned}
$$

By comparing equations (28) and (35) it follows that the two different approaches give the same final criteria. Its greatest advantage is that all modes are studied with the same range. Residual modes are not neglected; their influence on the structure dynamic can be more or less important using $\gamma$. Furthermore, the expression inside (28) and (35) has a physical meaning: it is the mechanical energy transmitted for the ith mode divided by the maximal mechanical energy that could be received. 


\section{OPTIMAL LOCATION OF SENSORS}

The optimal locations of sensors are determined in the same way than the optimal locations of actuators.

\section{Maximization of the system output}

When the system is released from the initial state $\{x(0)\}=\left\{x_{0}\right\}$, as when it is subjected to a persistent disturbance,(Hac and Liu, 1993) has shown that maximizing the system output $\int_{0}^{\infty}\{y\}^{T}\{y\} d t$ (for desired modes) yields maximizing the gramian observability matrix defined by :

$$
W_{o}=\int_{0}^{\infty} e^{[A]^{T} t}[C]^{T}[C] e^{[A] t} d t
$$

and $W_{o}$ tends to a diagonal form (for displacement sensors): $i=1, \ldots N$

$$
\left(W_{o}\right)_{i i}=\left(W_{o}\right)_{i+N, i+N}=\sum_{j=1}^{N_{s}} \frac{c_{j i}^{2}}{4 \zeta_{i} \omega_{i}}=\frac{1}{4 \zeta_{i} \omega_{i}} \sum_{j=1}^{N_{s}} c_{j i}^{2}
$$

Each diagonal term $\left(W_{o}\right)_{i i}$ corresponds to the maximization of the output energy $J_{i}$ for the ith mode, obtained if we consider the state equation reduced to the ith mode:

$$
\begin{gathered}
{\left[\begin{array}{c}
\omega_{i} \dot{\alpha}_{i} \\
\ddot{\alpha}_{i}
\end{array}\right]=\left[\begin{array}{cc}
0 & \omega_{i} \\
-\omega_{i} & -2 \zeta_{i} \omega_{i}
\end{array}\right]\left[\begin{array}{c}
\omega_{i} \alpha_{i} \\
\dot{\alpha}_{i}
\end{array}\right]+\left[\begin{array}{c}
{[0]} \\
+\left[b_{i l}\right]
\end{array}\right]} \\
\{y\}=\left[C_{i}\right]\left[\begin{array}{c}
0 \\
\dot{\alpha}_{i}
\end{array}\right] \quad J=\int_{0}^{\infty}\{y\}^{T}\{y\} d t
\end{gathered}
$$

Consequently, if the ith eigenvalue of $W_{o}$ is small, it means that the ith mode will not be well observed. To avoid this case, (Hac and Liu, 1993), (Baruh, 1992) suggest to find the sensors locations by maximizing a measure of $W_{o}$, like:

$$
\operatorname{trace}\left(W_{o}\right) *\left(\operatorname{det}\left(W_{o}\right)^{1 / 2 N}\right) \quad \min _{i}\left(W_{o}\right)_{i i}
$$

These criteria take into account the eigenmodes globally. They can be used with the residual modes as for actuators, in order to maximize the given information for the $N$ first modes and minimize the information about the $N^{R}$ modes (in order to minimize the spillover effect).

\section{The modified criteria}

As we want to have a convenient information for the $N$ first eigenmodes we suggest maximizing the output energy $J_{i}$ for each mode i (each diagonal term of $W_{o}$ denoted $\left.\left(W_{o}^{U}\right)_{i i}\right)$ instead of maximizing the global system output, and minimizing the output energy of each residual mode (called $\left.\left(W_{o}^{R}\right)_{i i}\right)$ :

$$
\begin{gathered}
\text { maximize } \quad\left\{\left(W_{o}^{U}\right)_{11}, \ldots,\left(W_{o}^{U}\right)_{N N}\right\} \\
\text { minimize }\left\{\left(W_{o}^{R}\right)_{11}, \ldots,\left(W_{o}^{R}\right)_{N^{R} N^{R}}\right\}
\end{gathered}
$$

This optimization problem has $N_{s}$ optimization parameters (the locations of the $N_{s}$ sensors) and $N$ optimization functions. It can be simplified by considering the new optimization criteria:

$$
\operatorname{maximize}\left\{\min _{i=1, \ldots N}\left(W_{o}^{U}\right)_{i i}-\gamma \max _{i=1, \ldots N^{R}}\left(W_{o}^{R}\right)_{i i}\right\}
$$

where $\gamma$ is a weighting constant. As the components of $W_{o}$ have not the same range, solving this problem can induce the study of particular modes instead of each of them, and then the obtained locations will not be optimal. Hence, we decide to establish homogeneity between each term $\left(W_{o}\right)_{i i}$ by dividing each of them by its maximal value obtained if the ith mode is the specific mode to be measured. The optimization problem becomes to maximize the minimal value of the homogeneous components:

$$
\begin{gathered}
\min _{i=1, N} \frac{\left(W_{o}^{U}\right)_{i i}}{\max _{\text {locations }}\left(W_{o}^{U}\right)_{i i}}-\gamma \max _{i=1, N^{R}} \frac{\left(W_{o}^{R}\right)_{i i}}{\max _{\text {locations }}\left(W_{o}^{R}\right)_{i i}} \\
=\min _{i=1, N} \frac{\sum_{j}^{N_{s}} c_{j i}^{2}}{\max _{\text {locations }} \sum_{j=1}^{N_{s}} c_{j i}^{2}}-\gamma \max _{i=1, N^{R}} \frac{\sum_{j}^{N_{s}} c_{j i}^{2}}{\max _{\text {locations }} \sum_{j=1}^{N_{s}} c_{j i}^{2}} \\
\text { with } \forall i=1, \ldots N+N^{R} \quad 0 \leq \frac{\left(W_{o}\right)_{i i}}{\max _{\text {locations }}\left(W_{o}\right)_{i i}} \leq 1
\end{gathered}
$$

Then all modes are studied with the same range. The first quotient inside (44) represents the output energy measured for the ith mode divided by the maximal output energy which could be measured for the ith mode by the sensors.

\section{APPLICATIONS}

In this section, we aim to compare the different usual criteria of controllability and observability with one modified criteria. In order to make comparisons, we define two degrees. 


\section{A degree of controllability and a degree of observability}

We define the following degree of controllability for each mode $\left(i=1, . . N+N^{R}\right)$ :

$$
D E G C i=\frac{\sum_{j=1}^{N_{a}} b_{i j}^{2}}{\max _{\text {actuator }} b_{i 1}^{2}}
$$

It equals to the energy transmitted from the actuators to the structure for the ith mode divided by the maximal value energy obtained if the ith mode is optimally controlled by one actuator (if one actuator is located in order to optimally control this mode). When the $N$ first degrees are over $100 \%$ it means that each mode $(i=1, \ldots N)$ is better controlled than when it is specifically controled by an actuator.

Inversely, for residual modes, the objective is to minimize $D E G C i$ the higher possible.

In the same way, the following degree of observability is defined for each mode $i$ :

$$
D E G O i=\frac{\sum_{j=1}^{N_{s}} c_{j i}^{2}}{\max _{\text {sensor }} c_{1 i}^{2}}
$$

It equals to the output energy measured from the sensors for the ith mode divided by the maximal value of output energy obtained if the ith mode is optimaly measured by one sensor (if one sensor is located to optimally measure this mode). When the $N$ degrees are over $100 \%$, each mode is better measured than when it is specifically measured by a sensor. Of course, the opposite is searched for residual modes.

In the next subsections, two applications are presented. The first one consists in comparing several criteria for actuators locations. The second one consists in studying the influence of residual modes in the optimization procedure.

\section{First application: optimal location of piezoelectric actuators on a simply supported plate}

A simply supported plate is considered, equiped with $N_{a}$ piezoelectric actuators to locate. In order to consider only pure bending motion, each actuator is made up of a pair of piezoelectric materials attached symetrically. They are assumed to be perfectly bonded to the surface of the plate, and their thickness is assumed to be small compared to the plate thickness. The geometrical and mechanical properties of the system are detailed in tables 1 and 2 .

table 1: characteristics of the plate

\begin{tabular}{lcl}
\hline Length $(\mathrm{m})$ & 0.38 & \\
width $(\mathrm{m})$ & 0.3 & \\
thickness $(\mathrm{m})$ & $0.0031752 \mathrm{E}(\mathrm{Pa})$ & $20710^{9}$ \\
$\nu$ & 0.292 & \\
$\rho\left(\mathrm{kg} / \mathrm{m}^{3}\right)$ & 7870 \\
\hline
\end{tabular}

table 2: characteristics of the PZT 5A

\begin{tabular}{lc}
\hline Length $(\mathrm{m})$ & 0.019 \\
width $(\mathrm{m})$ & 0.015 \\
thickness $(\mathrm{m})$ & 0.001 \\
$\epsilon_{33}(\mathrm{~F} / \mathrm{m})$ & $1.510^{-8}$ \\
$e_{31}\left(\right.$ C. $\left.m^{-2}\right)$ & -7.209 \\
$e_{32}\left(\right.$ C. $\left.m^{-2}\right)$ & -7.209 \\
\hline
\end{tabular}

The plate is divided in 400 rectangular elements which correspond to the possible locations of actuators. As the structure has two symmetry axes, we only consider its quarter. In this study, the first seven eigenmodes are used and all residual modes are neglected.

Simulations are presented for piezolectric actuators. Exactly the same results would be obtained for piezoelectric sensors measuring velocity (Bruant and Proslier, 2002).

The four following criteria are compared using $D E G C i$ :

$$
\begin{aligned}
& \min _{i} \frac{\left(W_{c}\right)_{i i}}{\max _{\text {locations }}\left(W_{c}\right)_{i i}} \text { called MINL/LMAX } \\
& \operatorname{trace}\left(W_{c}\right) *\left(\operatorname{det}\left(W_{c}\right)\right)^{1 / 2 N} \quad \text { called } \quad \text { TRACE } * D E T \\
& \min _{i}\left(W_{c}\right)_{i i} \quad \text { called } L \\
& \prod_{i=1}^{N_{a}} \sigma_{i}^{2} \text { called } W A N G
\end{aligned}
$$

Results for different actuators numbers are shown figures 1 to 3 . Figure 1.a represents, for each configuration, the smallest value $D E G C i(i=1 \ldots N)$. The others plots represent the degree of controllability of each eigenmode.

By comparing results, the smallest value DEGi has the highest values for the criteria MINL/LMAX. This is because other criteria use matrice (gramian or singular) which have not homogeneous components. Consequently, some eigenmodes are favourite instead of others. For example, for TRACE*DET the 6th mode and also modes 3,4 and 5 have a degree of controllability higher than the modes 1, 2 and 7 . This is also the case for $\mathrm{L}$ : the 1st mode is very well controlled instead of modes 4 and 6 which are very badly controlled even if seven actuators are used. In 
the optimization of WANG, the worst controlled mode is the fourth. Hence, these criteria ensure avoiding non controllability area, but do not ensure homogeneous con- trollability for considered modes. For MINL/LMAX, the variations of the degrees DEGCi are homogeneous, and they are more than $300 \%$ using seven actuators.

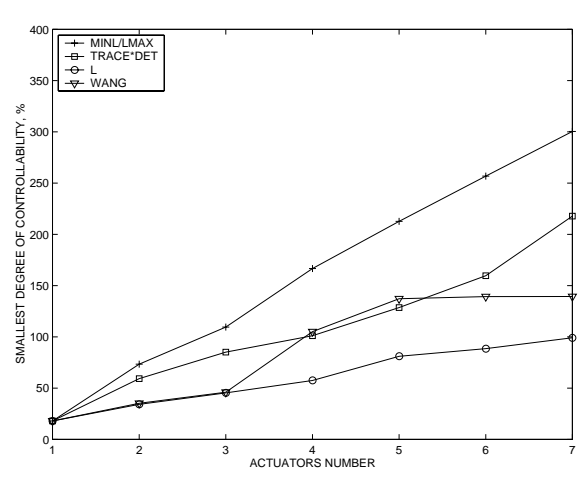

(a) Smallest degree of controllability

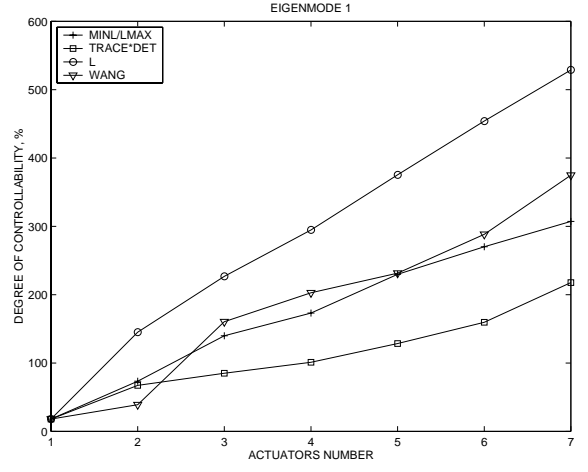

(b) First eigenmode

Figure 1 .

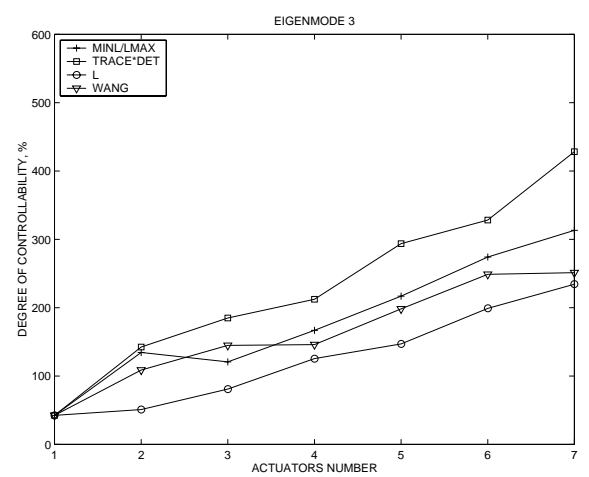

(a) eigenmode 3

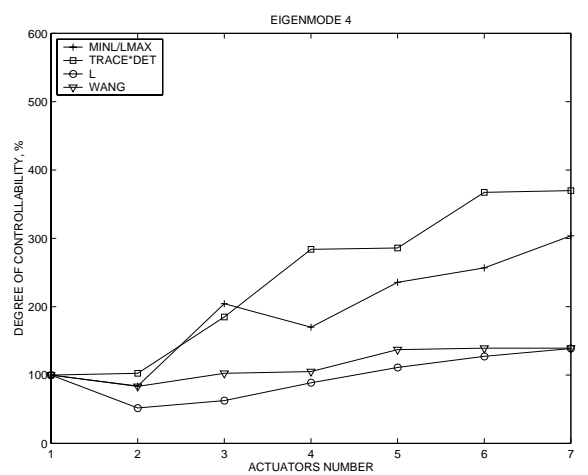

(b) eigenmode 4

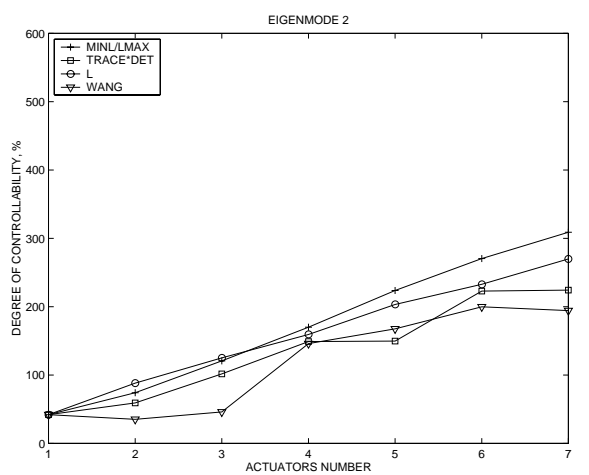

(c) Second eigenmode

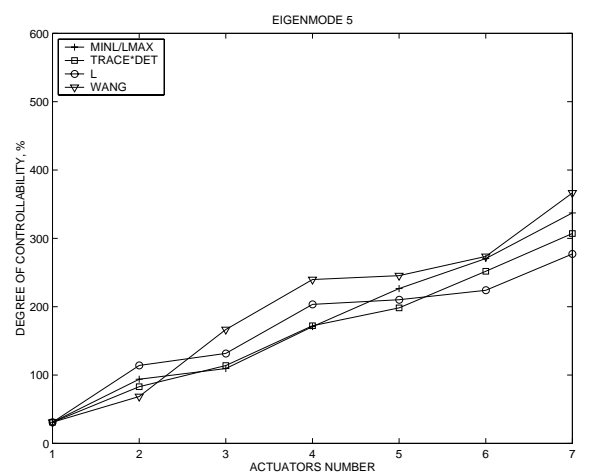

(c) eigenmode 5

Figure 2.

\section{About the number of actuators}

The controllability degree gives us information about the quality of the control for each mode. It can also be used to define the number of actuators needed. When the smallest value $D E G C i$, in figure 3 , is over $100 \%$ it means that each mode is better controlled than when it is specifically controlled by an actuator. Consequently, we can choose the value $100 \%$ as criteria for the optimal number of actuators. From this point of view, the number of actuators needed is for MINL/LMAX 3, even though TRACE*DET and WANG need 4 actuators and L needs 7 actuators.

Furthermore, the smallest value DEGCi is over $200 \%$ for MINL/LMAX using 5 actuators. Then, when a robust control system which ensures good controlability (over $100 \%$ for each mode) is necessary even if one actuator is failing, we will use this configuration. For the other criteria, it is not possible to use only 5 actuators located optimaly.

In this application, we have not considered the residual modes. In fact, for better optimal configuration of actuators, it should be necessary to take into account them. In our modal approach, it would consist in adding a sufficient number of residual modes. The use of the modal basis gives us a phyiscal meaning of gramian matrix.

In the next subsection, the residual modes are used to locate the sensors. 


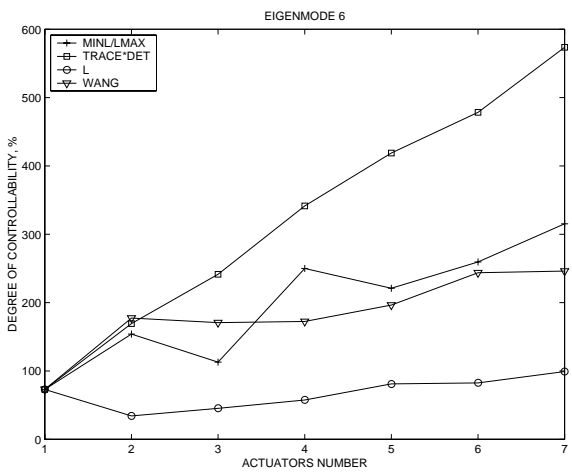

(a) eigenmode 6

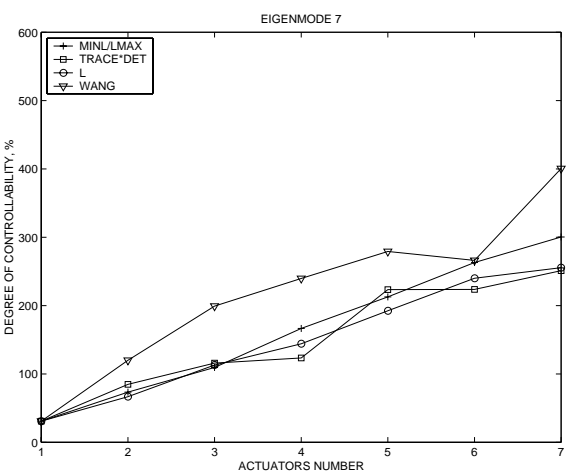

(b) eigenmode 7

Figure 3.

\section{Second application: optimal location of point sensors on a slender uniform beam}

As it was explained previoulsy, residual modes can be dangerous in active control, leading to instabilities. The used output sensors contain informations on motion on all modes instead of only the needed motion of the $N$ first modes. In the same way, the actuators actuate all modes of the real structure, and consequently can excite residual modes.

In order to illustrate the influence of residual modes in the optimization procedure, we consider here a simply supported uniform beam described in (Hac and Liu, 1993). In this case, an analytical analysis can be easily developed, and as the structure is symmetric, only the first middle of the beam is studied and divided in 50 possible point sensors locations. The observability gramian is equal to:

$$
\left(W_{o}\right)_{i i}=\frac{1}{2 \rho A l \zeta_{i} \omega_{i}}\left(\sum_{j=1}^{N_{S}} \sin ^{2} \frac{i \pi x_{j}}{l}\right)
$$

where $\rho A$ is the mass density, $x_{j}$ the location of the jth sensor and $l=1 \mathrm{~m}$, the beam length. We want to precisely measure the displacement of the first six modes while avoiding residual modes: modes 7, 8, 9 and 10 .

The results are also available for point actuators.

The considered criteria is defined in (44). it was used for different sensors quantity, with $\gamma=0$ and $\gamma=1$. Results are shown in tables 3 and 4 . For each case, the smallest degree of observability for needed modes (SDEGOi) and the highest degree of observability for residual modes $\left(H D E G O i^{R}\right)$ are presented. To avoid measure of residual modes, $H D E G O i^{R}$ has to be minimal, and to have good information about the first 6 modes, $S D E G O i$ has to be maximal, with $H D E G O i^{R}$ less than $S D E G O i$. table 3: results for $\gamma=0$

\begin{tabular}{lcc}
\hline sensors numbers & SDEGOi & HDEGOi \\
\hline 1 & $18 \%$ & $96 \%$ \\
2 & $93 \%$ & $100 \%$ \\
3 & $167 \%$ & $144 \%$ \\
4 & $221 \%$ & $325 \%$ \\
\hline
\end{tabular}

table 4: influence of the residual modes: $\gamma=1$

\begin{tabular}{lcc}
\hline sensors number & $S D E G O i$ & $H D E G O i^{R}$ \\
\hline 1 & $13,5 \%$ & $34 \%$ \\
2 & $75 \%$ & $74 \%$ \\
3 & $116 \%$ & $82 \%$ \\
4 & $191 \%$ & $136 \%$ \\
\hline
\end{tabular}

First of all, it can be seen from these results that forgetting residual modes in the optimization procedure $(\gamma=0)$ can be dangerous: except when there are 3 sensors, the maximal value $H D E G O i^{R}$ is over the minimal value $S D E G O i$. It means that one residual mode is better observed than a needed mode. Consequently, the sensors information can be strongly disturbed, inducing spillover effect.

When residual modes are taken into account in the optimization criteria, $H D E G O i^{R}$ is smallest than $S D E G O i$ except when there is one sensor. Comparing results for $\gamma=0$ and for $\gamma=1$ show that the value of each degree decreases between $\gamma=0$ and $\gamma=1$. When $\gamma=1$, the optimization procedure has to adjust the sensors locations obtained for $\gamma=0$ in order to avoid measurement of residual modes.

These kind of simulations can also help us to define the number of needed sensors. A good compromise between needed modes and residual modes seems to be obtained by using 3 sensors: each needed modes is better measured than each residual modes $\left(S D E G O i>H D E G O i^{R}\right)$. In an other part, the degrees of observability of the six first 
modes are over $100 \%$ and those of each residual modes is less than $100 \%$.

\section{CONCLUSION}

In this paper, the problem of sensors and actuators locations is considered. We propose for each optimization problem a modified optimization criteria derived from usual approaches, ensuring good observability and controllability of each mode of the structure, and taking into account residual modes which have to be less observable and controllable.

Their efficiency are shown by comparing them with classical criteria, especially when the number of active elements is varying. The results show how important is the choice of the optimization criteria for the effectiveness of the control. They also give us some help to define the optimal sensors and actuators number. The great advantages of these criteria are their computational simplicity, their non-dependance with the external disturbances and with the applied control law.

This methodology was used here considering actuators and sensors independently. It could be studied for collocated active elements. In the same way, same developpements could be done for active elements shape.

\section{REFERENCES}

Arbel, A. (1981). Controllability measures and actuator placement in oscillatory systems. International Journal of Control, 33 (3):565-574.

Baruh, H. (1992). Placement of sensors and actuators in structural control. Control and Dynamic Systems, 52:359-390.

Bruant, I., Coffignal, G., Léné, F., and Vergé, M. (2001). A methodology for determination of piezoelectric actuator and sensor location on beam structures. J. Sound Vibr., 243(5):862-882.
Bruant, I. and Proslier, L. (2002). Optimal location and number of sensors and actuators in active control. In 7-12 April, Come, I., editor, 3rd World Conf. on Structural Control.

Collet, M. (1999). Shape optimization of piezoelectric sensors dealing with spill-over instabilities. Proceedeings of Active 99, 2:1125-1134.

Devasia, S., Meressi, T., Paden, B., and Bayo, E. (1993). Piezoelectric actuator design for vibration suppression : plavement and sizing. Journal of Guidance, Control and Dynamics, 16 (5):859-864.

Dhingra, A. and Lee, B. (1995). Multiobjective design of actively controlled structures using a hybrid optimization method. International Journal for Numerical Methods in Engineering, 38:3383-3401.

Gaudiller, L. and Hagopian, J. D. (1996). Active control of flexible structures using a minimum number of components. Journal of Sound and Vibration, 193 (3):713-741.

Hac, A. and Liu, L. (1993). Sensor and actuator location in motion control of flexible structures. Journal of Sound and Vibration, 167 (2):239-261.

Kondoh, S., Yatomi, C., and Inoue, K. (1990). The positioning of sensors and actuators in the vibration control of flexible systems. JSME International Journal, srie 3, 33 (2):145-152.

Preumont, A. (1999). Vibration control of active structures. Kluwer Academic Publishers.

Wang, Q. and Wang, C. (2001). A controllability index for optimal design of piezoelectric actuators in vibration control of beam structures. Journal of Sound and Vibration, 242 (3):507-518.

Yang, S. and Lee, Y. (1993). Optimization of noncollocated sensor/actuator location and feedback gain in control systems. Journal of Smart Materials Structures, 2:96-102. 